\title{
Construção e validação de cartilha para cuidados paliativos domiciliares após alta hospitalar
}

Construction and validation of a booklet for home palliative care after hospital discharge Elaboración y validación de cartilla para cuidados paliativos domiciliarios después del alta hospitalaria

Francine Regazolli Ribeiro da Silva ${ }^{1}$ id https://orcid.org/0000-0002-7360-1320

Rosana Aparecida Pereira ${ }^{1}$ iD https://orcid.org/0000-0001-9389-3300

Ana Carolina de Souza ${ }^{1}$ id https://orcid.org/0000-0003-4126-4224,

Fernanda Raphael Escobar Gimenes ${ }^{1}$ (D) https://orcid.org/0000-0002-5174-112X

Giovana Paula Rezende Simino ${ }^{1}$ id https://orcid.org/0000-0002-9814-3004

Carina Aparecida Marosti Dessote ${ }^{1}$ iD https://orcid.org/0000-0002-5521-8416

Angelina Lettiere-Viana ${ }^{1}$ id https://orcid.org/0000-0002-4913-0370

Fabiana Bolela ${ }^{1}$ id hitps://orcid.org/0000-0003-1199-6205

Como citar:

Silva FR, Pereira RA, Souza AC, Gimenes FR, Simino

GP, Dessote CA, et al. Construção e validação de cartilha para cuidados paliativos domiciliares após alta hospitalar. Acta Paul Enferm. 2022;35:eAPE028112.

DOI

http://dx.doi.org/10.37689/acta-ape/2022A002812

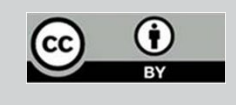

Descritores

Cuidados paliativos; Cuidado domiciliar; Educação em saúde; Estudos de validação

\section{Keywords}

Health education; Home care services; Palliative care; Validation study

Descriptores Cuidados paliativos; Educación en salud; Estudio de validación; Servicios de atención de salud a domicilio

Submetido 28 de Setembro de 2020 Aceito 23 de Março de 2021

\section{Autora correspondente \\ Fabiana Bolela}

E-mail: fbolela@usp.br

Editor Associado (Avaliação pelos pares): (https://orcid.org/0000-0002-6243-6497) Escola Paulista de Enfermagem, Universidade Federal de São Paulo, São Paulo, SP, Brasil

\section{Resumo}

Objetivo: Construir e validar cartilha educativa para cuidados paliativos domiciliares após a alta hospitalar.

Métodos: Estudo metodológico desenvolvido em três etapas: levantamento bibliográfico, construção da cartilha educativa e validação do material por especialistas em cuidados paliativos e cuidadores de pacientes. 0 processo de validação foi realizado por 8 especialistas e 12 representantes do público-alvo, selecionados por conveniência. Considerou-se o Índice de Validade de Conteúdo mínimo de 0,80, para validação de conteúdo e concordância mínima de 75\% para validação de aparência.

Resultados: A cartilha intitulada "Eu cuido, nós cuidamos - cuidados domiciliares a pacientes em cuidados paliativos" é composta por 28 páginas. Na validação de conteúdo pelos especialistas, o IVC global obtido foi 1,0 e a cartilha foi aprovada por unanimidade pelos representantes do público-alvo, com índice de concordância 1,0.

Conclusão: A cartilha educativa foi validada quanto ao conteúdo e aparência, podendo ser utilizada por cuidadores na realização de cuidados paliativos domiciliares e por enfermeiros para as orientações na alta hospitalar.

\section{Abstract}

Objective: To construct and validate an educational booklet for home palliative care after hospital discharge.

Methods: This is a methodological study developed in three stages: bibliographic survey, booklet construction and material validation by palliative care experts and patient caregivers. The validation process was carried out by 8 experts and 12 target audience representatives, selected for convenience. The Minimum Content Validity Index of 0.80 was considered for content validation and minimum agreement of $75 \%$ for appearance validation.

Results: The booklet "Eu cuido, nós cuidamos - cuidados domiciliares a pacientes em cuidados paliativos" consists of 28 pages. In the content validation by experts, the global CVI obtained was 1.0 and the booklet was unanimously approved by the target audience representatives, with agreement index of 1.0.

Conclusion: The educational booklet was validated regarding content and appearance, and can be used by caregivers in the performance of home palliative care and by nurses for guidance at hospital discharge.

\section{Resumen}

Objetivo: Elaborar y validar cartilla educativa para cuidados paliativos domiciliarios después del alta hospitalaria. 
Métodos: Estudio metodológico desarrollado en tres etapas: recopilación bibliográfica, elaboración de cartilla educativa y validación del material por especialistas en cuidados paliativos y cuidadores de pacientes. El proceso de validación fue realizado por ocho especialistas y 12 representantes del público destinatario, seleccionados por conveniencia. Se consideró el Índice de Validez de Contenido mínimo de 0,80 para la validación de contenido, y concordancia mínima de 75 \% para validación de apariencia.

Resultados: La cartilla titulada "Yo cuido, nosotros cuidamos: cuidados domiciliarios a pacientes en cuidados paliativos" está compuesta por 28 páginas. En la validación de contenido por los especialistas, el IVC global obtenido fue 1,0 y la cartilla fue aprobada por unanimidad por los representantes del público destinatario, con un índice de concordancia de 1,0.

Conclusión: La cartilla educativa fue validada respecto al contenido y apariencia y puede ser utilizada por cuidadores en la realización de cuidados paliativos domiciliarios y por enfermeros para dar instrucciones en el alta hospitalaria.

\section{Introdução}

Cuidado Paliativo (CP) é o cuidado holístico ativo a indivíduos de todas as idades, com sério sofrimento relacionado à saúde devido à doença grave e, especialmente, àqueles próximos ao fim de vida. Tem por objetivo melhorar a qualidade de vida de pacientes, suas famílias e cuidadores. ${ }^{(1)}$

No mundo todo, 53 milhóes de adultos necessitam de cuidados paliativos e, destes, $76 \%$ vivem em países de baixa ou média renda, como por exemplo, o Brasil. ${ }^{(2)}$ Dessa forma, discutir sobre estratégias para oferecer um cuidado seguro e eficaz no ambiente domiciliar pelo cuidador é essencial, tendo em vista o elevado número de indivíduos em tal condição.

Geralmente, após a alta hospitalar, o paciente necessita de cuidados seguros, de modo a eliminar os riscos de imperícias técnicas e, consequente redução do número de reinternaçóes e gastos com saúde, além de seu sofrimento e o de seus familiares. Considerando que, na maioria das vezes, o cuidador é um familiar leigo e sem conhecimento técnico, é necessária a capacitação e suporte educativo sobre os cuidados a serem desenvolvidos em domicílio, sobretudo se o paciente fizer uso de dispositivos de assistência. ${ }^{(3,4)}$

Orientaçôes básicas acerca da condição do paciente e de suas necessidades são essenciais para tornar possível a continuidade do cuidado prestado pelo cuidador familiar no domicílio e devem ser realizadas ainda durante a hospitalização. ${ }^{(3)}$

Entre as diversas tecnologias de informação, orientações e comunicação, materiais educativos, tais como, folhetos e cartilhas, contribuem com o processo educativo a cuidadores e familiares, favorecendo a adesão aos cuidados oferecidos aos pacientes, com o objetivo de melhorar sua qualidade de vida e, também, amenizar a insegurança e incertezas dos cuidadores. ${ }^{(5)}$
Assim, esse estudo teve como objetivo construir e validar uma cartilha educativa para cuidados paliativos domiciliares após a alta hospitalar.

\section{Métodos}

Estudo metodológico desenvolvido em três etapas: levantamento bibliográfico; elaboração do material educativo e validação do material por especialistas no assunto e representantes do público-alvo.

A cartilha foi construída de acordo com as recomendaçôes para produção e eficácia de materiais educativos, conforme as características: conteúdo, linguagem, ilustração, layout, motivação e cultura. ${ }^{(6)}$ No processo de construção da cartilha, foi realizado levantamento bibliográfico acerca dos cuidados domiciliares em manuais do Ministério da Saúde ${ }^{(7)}$ e da Academia Nacional de Cuidados Paliativos (ANCP), ${ }^{(8)}$ artigos nacionais e internacionais e livro texto de enfermagem. ${ }^{(9)}$

A coleta de dados foi realizada de dezembro de 2018 a maio de 2019, nas unidades de internação de um hospital universitário do interior paulista. Trata-se de instituição de média complexidade com 50 leitos e, destes, 10 são exclusivos para a internação de pacientes em cuidados paliativos.

A fim de incluir na cartilha temas relevantes e condizentes com as necessidades do seu público-alvo, o conhecimento prévio e a opiniáo de familiares e cuidadores de pacientes em cuidados paliativos acerca do cuidado domiciliar foram investigados mediante a realização de entrevistas.

A etapa de coleta de dados com os cuidadores contou com uma amostra consecutiva e não probabilística constituída por 10 cuidadores, com idade igual ou superior a 18 anos, que estavam acompanhando o paciente durante a internaçáo. 
Os cuidadores foram convidados a participar do estudo e esclarecidos sobre seus objetivos. Após o aceite, os cuidadores eram encaminhados à uma sala privativa e dava-se início a leitura do Termo de Consentimento Livre e Esclarecido (TCLE). Após a concordância e assinatura do TCLE pelo participante, as entrevistas eram iniciadas utilizando um roteiro estruturado com questóes que abordavam: capacidade do paciente para a realização do autocuidado; necessidade de auxílio para a administração de medicamentos no domicílio; uso de dispositivos para alimentação; características das eliminaçóes (espontânea ou não) e necessidade de uso de fraldas ou coletores; presença de estomas; grau de mobilidade do paciente e necessidade de dispositivos assistivos e sugestôes de conteúdo para compor a cartilha.

O roteiro foi construído pela pesquisadora principal e submetido a três especialistas para a validação aparente e de conteúdo. As entrevistas tiveram duração média de 15 minutos.

Após a definição do conteúdo da cartilha, foi realizada sessão de fotos no laboratório de baixa fidelidade da Escola de Enfermagem de Ribeirão Preto, da Universidade de São Paulo, com a participação de três voluntárias (duas representando as cuidadoras e uma representando a paciente), com o objetivo de obter imagens ilustrativas relacionadas ao conteúdo. Em seguida, o trabalho de design e diagramação foi realizado por profissional especialista em comunicação e publicidade e foi criada a primeira versão da cartilha.

Para validação da cartilha, usou-se o conceito de validade de conteúdo e aparência, ou seja, julgamento que busca medir a adequação dos itens de avaliação com relação ao conteúdo, além da concordância entre os especialistas. ${ }^{(10)}$

A carta convite aos especialistas foi enviada por endereço eletrônico, juntamente com o TCLE, a primeira versão da cartilha e seu protocolo de julgamento e um instrumento para caracterizaçáo sociodemográfica. Foi conferido aos especialistas um prazo de 30 dias para a avaliaçáo do material, preenchimento dos instrumentos e devolução à pesquisadora. Era facultativo aos especialistas acrescentar no protocolo sugestôes ou comentários sobre o material.

Foram convidados 10 profissionais com experiência assistencial, docência ou pesquisa, na área de cuidados paliativos e educação em saúde.
Entretanto, apenas oito responderam, aceitando participar. A busca por especialistas ocorreu por conveniência, via indicação de profissionais da área de cuidados paliativos e o número de especialistas inseridos no estudo foi considerado adequado. ${ }^{(11)}$

Um protocolo de julgamento foi adaptado para a validação do conteúdo e aparência da cartilha pelos especialistas e os 21 itens foram estruturados em tópicos: conteúdo, linguagem, ilustraçôes, layout, motivação e cultura. ${ }^{(12)}$ Os especialistas procederam à análise correspondente à relevância de cada item, (1-irrelevante, 2-pouco irrelevante, 3-relevante, 4-extremamente relevante). ${ }^{(13)}$ Ademais, o instrumento constava de espaço para comentários e sugestóes. Posteriormente, a cartilha foi submetida à edição para incorporaçáo das recomendaçóes e sugestóes dos especialistas, resultando na segunda versão.

Para a seleção de representantes do público-alvo para a avaliação de conteúdo e aparência, adotou-se os seguintes critérios de inclusão: ser alfabetizado e não possuir alteraçóes visuais que comprometessem a leitura da cartilha no momento.

Foram entregues aos participantes a segunda versão da cartilha educativa e o TCLE, sendo esclarecidos sobre o estudo e solicitada a assinatura do termo, em duas vias. Para avaliação da cartilha educativa pelos representantes do público-alvo, foi aplicado instrumento adaptado do Suitability Assessment of Materials (SAM), versão traduzida e adaptada para o português, ${ }^{(14)}$ contendo 15 itens. Cada participante foi instruído a realizar a leitura da cartilha, analisar o texto e as imagens e, em seguida, preencher o instrumento de avaliação, assinalando respostas que variavam de 0-não adequado, 1-parcialmente adequado, 2-totalmente adequado. Após a somatória de todos os itens e cálculos sugeridos, o material era classificado como, superior, adequado ou não-aceitável.

Ao aceitar participar do estudo, os representantes do público-alvo eram encaminhados à uma sala privativa, onde permaneciam sozinhos para a realização da leitura da cartilha por 15 minutos. Após esse tempo, a pesquisadora permanecia na sala durante o preenchimento do instrumento de avaliação, pelo participante, e nesse momento, dúvidas eram esclarecidas e o instrumento de avaliação e uma via do TCLE, recolhidos. 
Foi realizada análise descritiva dos dados referentes à caracterização dos especialistas e representantes do público-alvo. Para a verificação da validade de conteúdo da cartilha, foi utilizado o Índice de Validade de Conteúdo (IVC): calculou-se o Item-level Content Validity Index (I-CVI) referente a cada item do instrumento e o IVC global. Considerou-se o índice igual ou superior a 0,80 como desejável na validação do conteúdo. ${ }^{(10)}$

A análise estatística de concordância, segundo cada item do instrumento, foi realizada por meio da adequação do ajustamento das proporçóes dos especialistas que concordaram com a pertinência da cartilha educativa. Para tanto, empregou-se o teste Binominal, sendo pertinente o valor p igual ou superior a 0,85 . Para essa análise, o nível de significância adotado foi de $5 \%$.

O projeto foi aprovado pelo Comitê de Ética em Pesquisa (CEP) da Escola de Enfermagem de Ribeirão Preto, sob o parecer número 2.921.604, conforme a resolução 466/2012.(15)

\section{Resultados}

A versão final da cartilha educativa tem em sua dimensão 190x210mm (fechada) e 28 páginas de miolo (impressas frente e verso). Os temas foram agrupados por similaridade e adaptados para linguagem popular. Ao todo o conteúdo da cartilha foi composto por 15 tópicos acerca de cuidados gerais com o paciente sob cuidados paliativos e um tópico sobre o cuidado com o cuidador. A cartilha recebeu o título "Eu cuido, nós cuidamos - cuidados domiciliares a pacientes em cuidados paliativos".

A elaboração da primeira versão da cartilha partiu do levantamento bibliográfico acerca dos conteúdos relevantes para compor a cartilha e das sugestôes apontadas pelos cuidadores durante as entrevistas. As principais dúvidas e sugestóes dos cuidadores estáo apresentadas no quadro 1.

Ao todo, oito profissionais atuaram como especialistas na validação da cartilha, sendo seis enfermeiros, uma nutricionista e uma farmacêutica. No que se refere à titulação dos profissionais, seis são especialistas em cuidados paliativos e dois são mestres em outra área de conhecimento. Três atuam como do-
Quadro 1. Síntese de dúvidas e sugestões dos cuidadores entrevistados

\begin{tabular}{|l|l|}
\hline $\begin{array}{l}\text { Número de } \\
\text { cuidadores }\end{array}$ & Dúvidas e sugestões manifestadas \\
\hline 7 & $\begin{array}{l}\text { Dúvidas sobre o manuseio da sonda nasoentérica (administração de dieta, } \\
\text { medicamentos e água) }\end{array}$ \\
\hline 4 & Dúvidas sobre como cuidar dos estomas (traqueostomia e colostomia) \\
\hline 3 & Sugestão: inserir conteúdo sobre como oferecer o banho no leito \\
\hline 2 & Dúvidas sobre o que fazer quando a sonda nasoentérica é deslocada \\
\hline 1 & $\begin{array}{l}\text { Sugestão: inserir conteúdo sobre como transferir o paciente da cama para a } \\
\text { cadeira e da cadeira para a cama }\end{array}$ \\
\hline 1 & $\begin{array}{l}\text { Sugestão: inserir conteúdo sobre como deve ser o ambiente domiciliar para o } \\
\text { cuidado ao paciente }\end{array}$ \\
\hline
\end{tabular}

centes, ministrando aulas relacionadas a cuidados paliativos, cinco são profissionais que atuam diretamente na assistência a pacientes em cuidados paliativos. Ainda, para a validação da cartilha, participaram 12 representantes do público-alvo, em sua maior parte, filhos $(5 ; 41,6 \%)$, adultos $(9 ; 75 \%)$, com ensino médio completo $(4 ; 33,3 \%)$, sem vínculo empregatício formal $(9 ; 75 \%)$ e com renda de 2 salários mínimos $(6 ; 50 \%)$. Os itens avaliados e seus respectivos valores dos IVCs, se encontram detalhados na tabela 1.

Tabela 1. Concordância dos juízes aos itens da cartilha

\begin{tabular}{|c|c|c|c|}
\hline Item & $\mathrm{n}(\%)$ & $\mathrm{I}-\mathrm{CVI}{ }^{*}$ & $p$-valuet \\
\hline \multicolumn{4}{|l|}{ Conteúdo } \\
\hline $\begin{array}{l}0 \text { conteúdo atende uma possível situação de treinamento } \\
\text { do cuidador. }\end{array}$ & $8(100)$ & 1 & 1 \\
\hline Os títulos/ subtítulos são divididos de forma coerente. & $8(100)$ & 1 & 1 \\
\hline Os trechos em destaque merecem estar em destaque. & $8(100)$ & 1 & 1 \\
\hline 0 conteúdo atende às necessidades do público alvo. & $8(100)$ & 1 & 1 \\
\hline 0 texto possui sequência lógica. & $8(100)$ & 1 & 1 \\
\hline 0 conteúdo é relevante para os cuidadores. & $8(100)$ & 1 & 1 \\
\hline Do ponto de vista científico, o conteúdo está correto. & $8(100)$ & 1 & 1 \\
\hline \multicolumn{4}{|l|}{ Linguagem } \\
\hline A redação é compatível com o público alvo. & $8(100)$ & 1 & 1 \\
\hline As frases são atrativas. & $8(100)$ & 1 & 1 \\
\hline O texto possui clareza e objetividade. & $8(100)$ & 1 & 1 \\
\hline \multicolumn{4}{|l|}{ Ilustração } \\
\hline As ilustrações relacionam-se adequadamente ao conteúdo. & $8(100)$ & 1 & 1 \\
\hline As ilustrações são compreensíveis. & $8(100)$ & 1 & 1 \\
\hline As legendas ajudam o cuidador a compreender a imagem. & $8(100)$ & 1 & 1 \\
\hline $\begin{array}{l}0 \text { número de imagens é suficiente para abordar } 0 \\
\text { conteúdo. }\end{array}$ & $8(100)$ & 1 & 1 \\
\hline \multicolumn{4}{|l|}{ Layout } \\
\hline 0 tamanho e fonte da letra facilitam a leitura. & $8(100)$ & 1 & 1 \\
\hline As cores na cartilha viabilizam a leitura. & $8(100)$ & 1 & 1 \\
\hline A disposição dos itens nas páginas é organizada. & $8(100)$ & 1 & 1 \\
\hline 0 número de páginas e o tamanho do material é adequado. & $8(100)$ & 1 & 1 \\
\hline \multicolumn{4}{|l|}{ Motivação } \\
\hline 0 conteúdo incentiva o cuidador a prosseguir com a leitura. & $8(100)$ & 1 & 1 \\
\hline A cartilha é clara e esclarece as dúvidas dos cuidadores. & $8(100)$ & 1 & 1 \\
\hline \multicolumn{4}{|l|}{ Cultura } \\
\hline $\begin{array}{l}\text { A cartilha é capaz de atender a diferentes perfis de } \\
\text { cuidadores. }\end{array}$ & $8(100)$ & 1 & 1 \\
\hline
\end{tabular}

*Percentual de concordância no item; I-CVI - Item-Level Content Validity Index; †Teste binomial 
Os tópicos "orientaçóes sobre o contato com animais domésticos" e "cuidados com o cuidador" foram sugeridos por três especialistas como relevantes para compor a cartilha. As sugestóes foram acatadas e os tópicos foram inseridos na cartilha.

No que se refere à avaliação realizada pelos representantes do público-alvo, houve unanimidade nas respostas de todos os participantes sobre a relevância dos itens avaliados na cartilha, ou seja, houve $100 \%$ de concordância em todos os itens avaliados. A única sugestão realizada por um participante foi sobre aumentar o tamanho da fonte adotada no texto. A sugestão foi acatada e com isso, definida a versão final da cartilha.

\section{Discussão}

Atualmente observa-se uma preferência por parte de pacientes e suas famílias para que os cuidados paliativos sejam realizados em seus domicílios. Essa tendência remete de volta às famílias a responsabilidade dos cuidados. Entretanto, para que essa preferência do paciente seja atendida é necessário que sua família tenha estrutura para o cuidado. ${ }^{(16)}$ Diante de um paciente com inúmeras necessidades, o familiar/ cuidador passa a exercer funçóes muitas vezes desconhecidas pela maioria deles. Logo, essas atividades podem gerar desgastes emocionais, físicos, sociais e econômicos impactando negativamente em sua saúde mental e qualidade de vida. ${ }^{(17)}$

Dessa forma, o uso de cartilhas educativas e a disponibilização de manuais no formato impresso ou online, possibilita que as orientaçôes de enfermagem sejam assimiladas para além do ambiente hospitalar, ampliando a compreensão das informaçôes e fortalecendo a aprendizagem para o cuidado. Além disso, o uso de materiais educativos em saúde é essencial para intensificar as orientaçôes fornecidas, sendo instrumentos viáveis, com linguagem simples e de grande apoio para os cuidados domiciliares. ${ }^{(18)}$

Por meio de busca bibliográfica na literatura nacional e internacional foi possível observar a escassez de estudos sobre construção e validação de materiais educativos direcionados a cuidadores. Em tal busca foi identificado um estudo recente realizado na região Sul do Brasil, que teve por objetivo elaborar uma cartilha para auxiliar os cuidadores de pacientes com doença incurável, no processo de orientação quanto aos principais cuidados, sintomas e informaçóes capazes de amenizar os momentos finais de vida, de modo a oferecer conforto e qualidade de vida ao paciente. Os autores percorreram a etapa de busca bibliográfica para fundamentação do conteúdo e a construção da cartilha, propriamente dita. Entretanto, não foi realizada a etapa de validação do material por especialistas ou representantes do público-alvo. ${ }^{(19)}$

A confecção de um material educativo tem como propósito contribuir com o desenvolvimento de habilidades, favorecendo a autonomia frente às atividades e decisões dos pacientes e familiares. ${ }^{(20)}$

Assim, no presente estudo, cada etapa percorrida para o alcance do objetivo proposto mostrou-se fundamental. Associar ao levantamento bibliográfico, acerca dos cuidados domiciliares, a coleta de informaçóes relevantes e condizentes com as necessidades dos cuidadores, foi primordial para a definição do conteúdo a ser incluído na cartilha, permitindo que o material possa, de fato, contribuir para a oferta de cuidados domiciliares aos pacientes (Figuras 1, 2 e 3).

Ainda, no processo de validação, a participação de representantes do público alvo é importante pois possibilita a opiniáo dos mesmos quanto ao conteúdo, linguagem e aparência, permitindo que o material seja adequado para a população para o qual se destina. ${ }^{(21)}$ Além disso, o público-alvo também aponta aspectos relevantes a serem incluídos, como pôde ser percebido no presente estudo. ${ }^{(22)}$

A opção por incluir fotografias relacionadas ao conteúdo teórico da cartilha foi por considerá-las mais atrativas. A ilustração adequada de um material educativo desperta o interesse do leitor em conhecer seus conteúdos por ter suas formas criativas atreladas às evidências científicas atualizadas. ${ }^{(23)}$ Também, a adoção de uma linguagem simples e convidativa, facilita a compreensáo dos conteúdos e a atratividade pelo texto. ${ }^{(24)}$

A etapa final consistiu na validação do material construído. A participação de membros da equipe multiprofissional e não só da equipe de enfermagem 


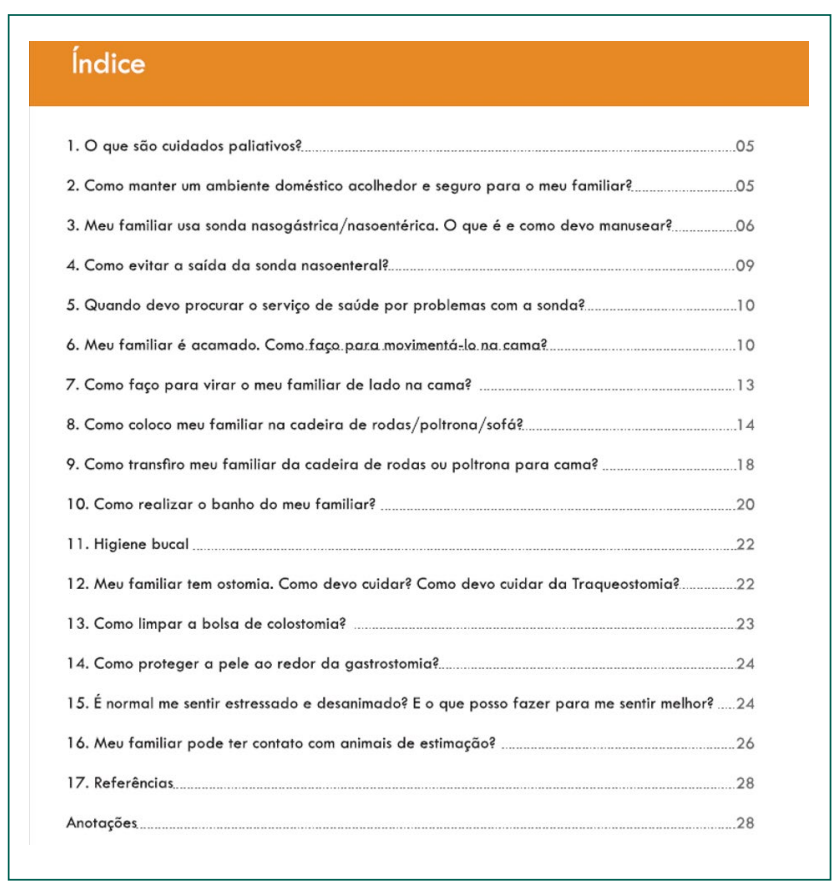

Figura 1. Índice da cartilha educativa "Eu cuido, nós cuidamos - cuidados domiciliares a pacientes em cuidados paliativos"

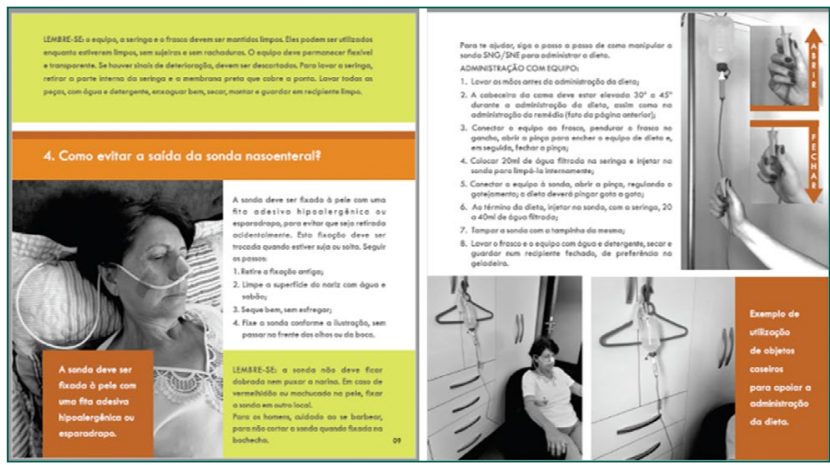

Figura 2. Algumas páginas da cartilha educativa "Eu cuido, nós cuidamos - cuidados domiciliares a pacientes em cuidados paliativos"

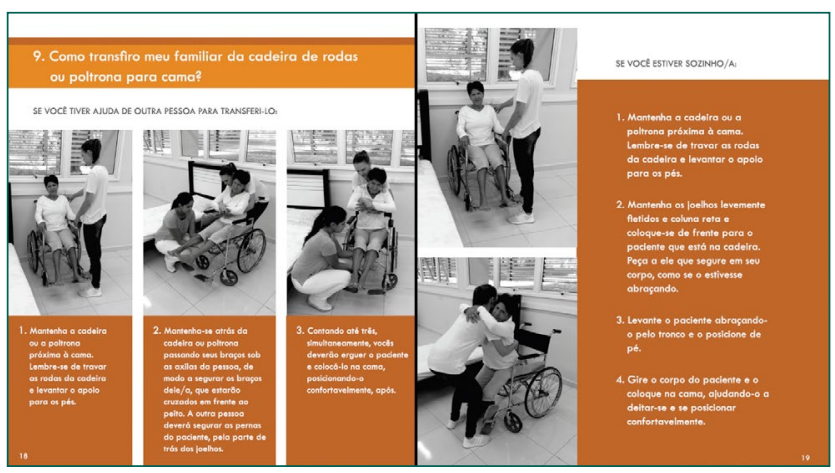

Figura 3. Algumas páginas da cartilha educativa "Eu cuido, nós cuidamos - cuidados domiciliares a pacientes em cuidados paliativos" no corpo de especialistas foi de grande importância para a avaliação da cartilha educativa, uma vez que no processo de construção de materiais educativos a soma das experiências e da criatividade permite a criação de materiais com melhores conteúdos e melhores formas de apresentação, facilitando o diálogo mesmo a distância com os pacientes e seus cuidadores. ${ }^{(24)}$ Embora não tenha sido realizada qualquer alteração significativa na versão inicial da cartilha, as sugestôes de inclusão de conteúdos relevantes foram acatadas.

Um especialista sugeriu a inclusão de um tópico sobre o convívio com animais de estimação. Tal sugestão foi acatada, considerando as recomendaçóes de profissionais e especialistas quanto aos benefícios do contato com os animais para os pacientes adultos e idosos. Uma publicação trimestral de um importante serviço de combate ao câncer, no Brasil, abordou o trabalho que tem sido desenvolvido, denominado Pet Terapia, enfatizando que a ciência comprova que o contato com os animais ajuda a liberar os chamados "hormônios do bem", aumentando a produção de endorfina (considerada um analgésico natural) e serotonina (que atua no cérebro regulando humor, sono e apetite) e reduzindo as taxas de cortisol. $^{(25)}$

Uma pesquisa realizada em hospital pediátrico privado da cidade de São Paulo, identificou que a companhia dos animais pode afastar a dor, a tristeza e o medo. Além de proporcionar sentimentos positivos, sensação de conforto e bem-estar. ${ }^{(26)}$ Além disso, a Terapia Assistida por Animais (TAA) é uma intervenção direcionada, individualizada e com critérios específicos em que o animal é parte integrante do processo do tratamento e tem por objetivo, desenvolver e melhorar aspectos sociais, físicos, emocionais e cognitivos, sendo desenvolvida junto com profissional da saúde. ${ }^{(27)}$

A TAA pode ser aplicada em várias faixas etárias e em diferentes contextos, tais como, hospitais, ambulatórios, casas de repouso, escolas, clínicas de fisioterapia e de reabilitação. Podem ser utilizados diversos tipos de animais que possam entrar em contato com os humanos sem proporcionar-lhes perigo, sendo que o cão é o mais utilizado, por sua natural afeição pelas pessoas, possibilidade de ades- 
tramento, cria respostas positivas ao toque e tem grande aceitação por parte das pessoas. ${ }^{(26)}$

Outro tópico sugerido por dois especialistas e acatado nesse estudo relaciona-se aos cuidados com o cuidador, em especial, no que se refere à sua saúde mental. Estudos apontam que os efeitos negativos sobre a saúde física e mental de cuidadores, são resultantes da interação entre a exposição do cuidador aos estressores, sua vulnerabilidade e seus recursos psicológicos e sociais. ${ }^{(28)} \mathrm{O}$ cansaço emocional caracterizado pela perda progressiva de energia, fadiga e esgotamento emocional, ocasionado pela assistência cotidiana prestada a pessoas que demandam cuidados, requerem intervençôes em diferentes níveis a fim de alcançar um suporte adequado. ${ }^{(29)}$

O processo de validação com avaliação de itens referentes ao objetivo, estrutura/apresentação e relevância é importante para que materiais educativos não possuam informaçóes equivocadas ou incompletas que possam induzir a população-alvo ao erro ou dificultar o entendimento da temática. ${ }^{(21)}$

As análises estatísticas permitiram identificar a unanimidade da concordância, tanto dos especialistas, quanto dos representantes do público-alvo ao material elaborado. Conforme observado na tabela 1 , os 18 itens foram avaliados pelos especialistas como adequados, com nível de concordância de $100 \%$, ou seja, o IVC global na validação pelos especialistas foi igual a 1,0 que é considerado padrão-ouro.

Tal resultado corrobora com outros estudos metodológicos realizados no Brasil. Um estudo que teve por objetivo validar o conteúdo de uma cartilha para cuidadores de crianças com hidrocefalia, obteve IVC entre 0,9 e $1,0 .{ }^{(30)}$ Outro estudo para validação de cartilha para prevenção de síndrome metabólica em adolescentes obteve IVC de 1,0 na maioria dos itens analisados. ${ }^{(31)}$ Dessa forma, o alcance de índices elevados constata a adequada validação do material quanto ao conteúdo e aparência, assim como ocorreu no presente estudo.

Uma limitação do estudo é que o material foi avaliado apenas por cuidadores de pacientes usuários do Sistema Único de Saúde, de modo que os resultados obtidos podem divergir da realidade de cuidadores de pacientes atendidos na rede privada de assistência à saúde.

\section{Conclusão}

Foi alcançado o objetivo do estudo em descrever a construção e validação da cartilha educativa para cuidados paliativos domiciliares após a alta hospitalar. O IVC global, alcançado na avaliação por especialistas e a total concordância entre os representantes do público-alvo conferem, respectivamente, a validade de conteúdo e de aparência da cartilha educativa em saúde intitulada "Eu cuido, nós cuidamos -cuidados domiciliares a pacientes em cuidados paliativos". Por meio da construção da cartilha educativa, os cuidadores de pacientes internados sob cuidados paliativos, receberão a cartilha na alta hospitalar para auxiliando-os nos cuidados domiciliares. Ainda, esse material contribuirá para a assistência do enfermeiro no planejamento e orientaçôes para a alta hospitalar, de modo a proporcionar autonomia e suporte ao cuidador. Nesse sentido, sugere-se a realização de estudo de aplicação, como também a realização de revisôes anuais do manual, com base no conhecimento científico mais atual.

\section{Colaborações}

Silva FRR, Pereira RA, Souza AC, Gimenes FRE, Simino GPR, Dessote CAM, Lettiere-Viana A e Bolela F contribuíram com a concepção do projeto, análise e interpretação dos dados, redação do artigo, revisão crítica relevante do conteúdo intelectual e aprovação da versão final a ser publicada.

\section{Referências}

1. Radbruch L, De Lima L, Knaul F, Wenk R, Ali Z, Bhatnaghar S, et al. Redefining Palliative Care-A New Consensus-Based Definition. J Pain Symptom Manage. 2020;60(4):754-64.

2. World Health Organization (WHO). Worldwide Palliative Care Alliance (WPCA). Global Atlas of Palliative Care. 2nd ed. Geneva: WHO; WPCA: 2020.

3. Souza IC, Silva AG, Quirino AC, Neves MS, Moreira LR. Perfil de pacientes dependentes hospitalizados e cuidadores familiares: Conhecimento e preparo para as práticas do cuidado domiciliar. Rev Min Enferm. 2014;18(1):164-72.

4. Freitas $A A$, Cabral IE. 0 cuidado à pessoa traqueostomizada: análise de um folheto educativo. Esc Anna Nery. 2008;12(1):84-9. 
5. Rocha EM, Paes RA, Sthal GM, Souza A. Cuidados paliativos: cartilha educativa para cuidadores de pacientes oncológicos. Clin Biomed Res. 2019;39(1):40-57.

6. Hoffmann T, Worrall L. Designing effective written health education materials: considerations for health professionals. Disabil Rehabil. 2004;26(19):1166-73. Review.

7. Brasil. Ministério da Saúde. Secretaria de Atenção à Saúde. Secretaria de Gestão do Trabalho e da Educação na Saúde. Guia prático do cuidador. Brasília (DF): Ministério da Saúde; 2008 [citado 2020 Mar 7]. Disponível em: http://bvsms.saude.gov.br/bvs/publicacoes/guia_ pratico_cuidador.pdf

8. Carvalho RT, Parsons HÁ, organizadores. Manual de cuidados paliativos. Ampliado e atualizado. 2a ed. São Paulo: ANCP; 2012.

9. Potter PA, Perry AG. Fundamentos de enfermagem. 9a ed. Rio de Janeiro: Elsevier; 2017.

10. Polit DF, Beck CT. The content validity index: are you sure you know what's being reported? Critique and recommendations. Res Nurs Health. 2006;29(5):489-97.

11. Miura CT, Gallani MC, Domingues GB, Rodrigues RC, Stoller JK. Cultural adaptation and reliability analysis of the Modified Dyspnea Index for the Brazilian culture. Rev Lat Am Enferm. 2010;18(5):1020-30.

12. Galindo Neto NM, Caetano, JA, Barros LM, Silva TM, Vasconcelos EM. First aid in schools: construction and validation of an educational booklet for teachers. Acta Paul Enferm. 2017;30(1):87-93.

13. DeVon HA, Block ME, Moyle-Wright P, Ernst DM, Hayden SJ, Lazzara DJ, et al. A psychometric toolbox for testing validity and reliability. J Nurs Scholarsh. 2007;39(2):155-64. Review.

14. Sousa CS, Turrini RN, Poveda VB. Translation and adaptation of the instrument "suitability assessment of materials" (sam) into portuguese. Rev Enferm UFPE Online. 2015;9(5):7854-61.

15. Brasil. Ministério da Saúde. Conselho Nacional de Saúde. Resolução nº 466, de 12 de dezembro de 2012. Brasília (DF): Ministério da Saúde; 2012 [citado 2020 Out 18]. Disponível em: http://bvsms.saude.gov.br/ bvs/saudelegis/cns/2013/res0466_12_12_2012.html

16. Balisa MF, Bousso RS, Spineli VM, Silva L, Poles K. Palliative care in the home: perceptions of nurses in the Family Health Strategy. Acta Paul Enferm. 2012;25(Spe2):13-8.

17. Oliveira MB, Souza NR, Bushatsky M, Dâmaso BF, Bezerra DM, Brito JA. Oncological homecare: family and caregiver perception of palliative care. Esc Anna Nery. 2017;21(2):e20170030.

18. Cruz FO, Ferreira EB, Vasques $\mathrm{Cl}$, Mata LR, Reis PE. Validation of an educative manual for patients with head and neck cancer submitted to radiation therapy. Rev Lat Am Enferm. 2016;24:e2706.
19. Rocha EM, Paes RA, Sthal GM, Souza A. Cuidados paliativos: cartilha educativa para cuidadores de pacientes oncológicos. Clin Biomed Res. 2019;39(1):40-57.

20. Souza LM, Morais RL, Oliveira JS. Direitos sexuais e reprodutivos: influências dos materiais educativos impressos no processo de educação em sexualidade. Saúde Debate. 2018;39(106):683-93.

21. Ximenes MA, Fontenele NA, Bastos IB, Macêdo TS, Galindo Neto NM, Caetano JA, et al. Construction and validation of educational booklet content for fall prevention in hospitals. Acta Paul Enferm. 2019;32(4):433-41.

22. Siqueira AF, Ferreira DS, Monteiro WF, Teixeira E, Barbosa IP. Validação de manual sobre prevenção do suicídio para universitários: falar é a melhor solução. Rev Rene. 2020;21:e42241.

23. Albuquerque AF, Pinheiro AK, Linhares FM, Guedes TG. Technology for self-care for ostomized women's sexual and reproductive health. Rev Bras Enferm. 2016;69(6):1164-71.

24. Varela Al, Rosa LM, Radünz V, Salum NC, Souza Al. Cartilha educativa para pacientes em cuidados paliativos e seus familiares: estratégias de construção. Rev Enferm UFPE Online. 2017;11(Supl 7):2955-62.

25. Instituto Nacional de Câncer José Alencar Gomes da Silva (INCA). Publicação trimestral do Instituto Nacional de Câncer José Alencar Gomes da Silva. Rede Câncer, 2016. Cãoterapia. Rio de Janeiro: INCA; 2016 [citado 2021 Set 27]. Disponível em: https://www.inca.gov.br/ sites/ufu.sti.inca.local/files/media/document/rrc-34-versao-integral. pdf

26. Vaccari AM, Almeida FA. The importance of pets' visit in recovery of hospitalized children. einstein (São Paulo). 2007;5(2):111-6.

27. Nobre MO, Krug FD, Capella SO, Ribeiro VP, Nogueira MT, Canielles $\mathrm{C}$, et al. Projeto pet terapia: intervenções assistidas por animais: uma prática para o benefício da saúde e educação humana. Expres Extensão. 2017;22(1):78-89.

28. Santos Junior AG, Santos FR, Pessalacia JD. Challenges for hospice care in primary health care: integrative review literature. Rev Enferm UFPE Online. 2016;10(7):2708-19.

29. Ruviaro MF, Bardagi MP. Síndrome de burnout e satisfação no trabalho em profissionais da área de enfermagem do interior do RS. Barbaroi. 2010;33:194-216.

30. Tavares PAJ, Hamamoto Filho PT, Ferreira AS, Avila MA. Construction and validation of educational material for children with hydrocephalus and their informal caregivers. World Neurosurg. 2018;114:381-90.

31. Moura IH, SilvaAF, RochaAE, Lima LH, Moreira TM, SilvaAR. Construction and validation of educational materials for the prevention of metabolic syndrome in adolescents. Rev Lat Am Enferm. 2017:25:e2934. 